\author{
Dr. Jürgen Bachmann \\ Schmerzmedizin - Orthopädie - Translationale Medizin \\ Sünsbruch 16, 45527 Hattingen, Deutschland \\ dza@dr-bachmann-hattingen.de
}

\title{
Akupunktur kann mehr!
}

Liebe LeserInnen und KollegInnen,

vor Ihnen liegt die letzte Ausgabe des 1. Coronajahrgangs. Ergänzend zu den News rund um die Akupunktur haben wir bereits in den letzten Heften - Sie werden es sicher bemerkt haben - die einschlägigen Nachrichten zu den Möglichkeiten der chinesischen Medizin und den Erfahrungen im Mutterland des Virus als eigenständige Rubrik unserer News gefasst. Möge sich die Notwendigkeit einer solchen Rubrik alsbald verlieren.

Was erwartet Sie im wissenschaftlichen Teil unseres Heftes? Unsere Serie von Aufarbeitungen des aktuellen Publikationsstandes konnte bereits für eine ganze Reihe einzelner Indikationen aufzeigen, dass nachweislich mehr Möglichkeiten gegeben sind, als in den 2 Diagnosen der Qualitätssicherungsvereinbarung des EBM oder der Leistungslegende der GOÄ formuliert wird. Wer sich für die Behandlung von Fertilitätsstörungen interessiert, findet in dieser Ausgabe Anregungen zur Behandlung und Argumentationshilfen für den Fall, dass sich das soziale Umfeld, der Kollegenkreis oder gar der Kostenträger skeptisch, ablehnend oder ignorant zeigen sollten.

Im Journal Club erscheint es als Highlight, dass in die Richtschnur für die Finanzierung von Gesundheitsleistungen durch den National Health Service (NHS) eine Empfehlung für die Anwendung von Akupunktur bei chronischen Schmerzen aufgenommen worden ist. A. Wiebrecht arbeitet allerdings heraus, wo der Haken liegt. Vom gleichen Autor stammt auch der Beitrag zur begrenzten Evidenz für chinesische Arzneitherapie bei COVID-19. T. Ots widmet sich einem systematischen Review zum Effekt von Akupunktur und Moxibustion bei chronischem Harnwegsinfekt.

Der Praxisteil widmet sich schwerpunktmäßig dem Schlaf bzw. den therapeutischen Möglichkeiten, wenn selbiger gestört ist. Diese weisen über Shen Men an Hand und Ohr hinaus: einleitend die Ergebnisse einer Internetrecherche zum aktuellen Stand der Publikationen durch M. Bijak, T. Ots berichtet über die Förderung des Schlafes anhand ausgewählter Punkte, A. Meng unter dem Aspekt der Punktekombinationen und möglichen Syndromdifferenzierung. Die phytotherapeutischen Möglichkeiten beleuchten C. Krassnik unter Einschluss westlicher Arzneipflanzen und $U$. Eberhardt im Hinblick auf die japanische Kampo.

Deutsche Zeitschrift für Akupunktur 2020 • 63 (4): 207

https://doi.org/10.1007/s42212-020-00330-z

(c) Springer Medizin Verlag GmbH, ein Teil von Springer Nature 2020
Auch unsere „Kolumne“ von Frau Kollegin Tauber-Bachmann ist dem Schlaf gewidmet!

Mit Fallberichten zur Akupunktur der Notfallmedizin, diesmal mit Beispielen aus Orthopädie und Schmerztherapie schließt T. Schockert seine Kurzserie ab.

Trotz der Pandemie hat es die Redaktion geschafft, den in der 1. Coronawelle abgesagten Termin der Redaktionskonferenz im September in Wien stattfinden zu lassen. Die dort erarbeitete Planung für die Schwerpunktthemen sei hier schon vorab umrissen, nicht zuletzt um Experten unserer Leserschaft für eine Mitarbeit an den genannten Schwerpunktheften zu gewinnen: Der erste Schwerpunkt des kommenden Jahres wird der Ohrakupunktur gehören, das zweite Heft die Behandlung von Erkrankungen des Auges fokussieren. Die weiteren Schwerpunkte widmen sich der Elektrostimulation, der Akupunkturanwendung beim älteren Patienten und einem vielgestaltigen Bild, dem sogenannten Fibromyalgiesyndrom.

Sie erinnern das Schwerpunktheft aus November 2019 und den darin enthaltenen Kommentar und Aufruf? Dem wird Rechnung getragen werden! Schließlich werden wir die Thematik um die Anwendung von Akupunktur in Notfallsituationen über die Ihnen in diesem Heft mit einem zweiten und letzten Beitrag vorliegende Aufarbeitung in Form von Fallberichten hinaus vertiefend betrachten. In der Anwendung liegen Besonderheiten: Die äußeren Bedingungen erlauben in der Regel nur eingeschränkte Lagerung, die Akutsymptomatik steht im Vordergrund und Aspekte der Ganzheitlichkeit oder auch eines biopsychosozialen Modells treten in den Hintergrund. Mit den Schlagworten Katastrophenhilfe, Battlefield Acupuncture und auch Gesundheitsprojekte der Entwicklungshilfe wird ein Feld umrissen, das über die leibliche Erfahrung und individuelle Therapie hinaus wesentliche politische Aspekte umfasst. Dies gilt auch für unser Vorhaben, einen Schwerpunkt der Frage zu widmen, wie für die Akupunktur die Rollenverteilung zwischen der ärztlichen und weiteren Berufsgruppen der medizinischen Versorgung bestimmt werden sollte.

Schließlich möchte ich hier Aufmerksamkeit auf die Darstellung von D. Irnich lenken: die brandneue (Muster-)Weiterbildungsordnung und das dazugehörige Kursbuch Akupunktur ein kleines Lehrstück aus der Berufs- und Gesundheitspolitik!

Ihr

Jürgen Bachmann

Interessenkonflikt. J. Bachmann gibt an, dass kein Interessenkonflikt besteht. 